\title{
Caffeinated Drinks, Alcohol Consumption, and Hangover Severity
}

\author{
Renske Penning, Lydia de Haan and Joris C. Verster \\ Utrecht University, Utrecht Institute for Pharmaceutical Sciences, Division of Pharmacology, Universiteitsweg 99, 3584 \\ CG Utrecht, The Netherlands
}

\begin{abstract}
This study examined the relationship between consumption of caffeinated beverages and alcohol, and effects on next day hangover severity. In 2010, a survey funded by Utrecht University was conducted among N=549 Dutch students. Beverages consumed on their latest drinking session that produced a hangover were recorded. Hangover severity was scored using the Acute Hangover Scale. No significant correlation between caffeine use and hangover severity was found. Subjects who mixed alcohol with colas consumed significantly more alcohol than those who drank alcohol alone $(\mathrm{p}=0.001)$, or mixed alcohol with energy drinks $(\mathrm{p}=0.001)$. Future studies with larger sample sizes should confirm these findings.
\end{abstract}

Keywords: Caffeine, alcohol, hangover, cola beverages, energy drinks.

\section{INTRODUCTION}

The alcohol hangover comprises the feeling of general misery experienced the day after an evening of excessive alcohol consumption. In fact, alcohol hangover is the most commonly reported adverse effect of excessive alcohol consumption [1]. The symptoms experienced during alcohol hangover vary within and between people, both in presence and severity [2]. Frequently reported symptoms include being tired, headache, weakness, nausea, thirst, and concentration problems. Alcohol hangovers have serious socioeconomic and health consequences such as absenteeism and presenteeism [3], and these consequences however are often underestimated [4].

Hence, research on 'cures' that may either prevent or ease alcohol hangovers is in its early stages and currently no effective treatment is available $[5,6]$. One of the main reasons for the lack of effective treatments is the incomplete knowledge on the pathology of alcohol hangover [2]. Until the pathogenesis of hangover is revealed, many 'cures' will be marketed on the internet without providing scientific evidence for their claim of being effective. In addition, drinkers sometimes experience to invent new ways to ease or prevent hangovers, and anecdotic 'evidence' of effectiveness can be found on many websites [6]. An example of these presumed hangover cures are caffeinated beverages, such as coffee, colas, and energy drinks.

Anecdotic evidence and surveys reveal that coffee is sometimes consumed during alcohol hangover, and internet searches show that pills containing aspirin combined with caffeine are popular to ease hangovers as well [6]. Aspirin is thought to relief headache, whereas caffeine is added to counteract fatigue and drowsiness. However, up to now the

*Address correspondence to this author at the Utrecht Institute for Pharmaceutical Sciences, Division of Pharmacology, Utrecht University, Universiteitsweg 99, 3584 CG, Utrecht, The Netherlands;

Tel: +31 30253 6909; E-mail: j.c.verster@uu.nl assumption that caffeine can prevent or ease alcohol hangovers has not been scientifically investigated. Sources of caffeine other than coffee, such as colas are frequently combined with alcoholic beverages. Although no claims have been made for colas, recent surveys report that energy drinks are sometimes consumed for the purpose of preventing or treating alcohol hangover [7,8].

It is not expected that other ingredients of cola beverages or energy drinks (i.e. sugar, taurine, and B Vitamins) interfere with or enhance potential effects of caffeine on hangover symptoms and their severity. For example, Ylikahri et al. [9] showed that administration of sugars (fructose or glucose) had no significant effect on the rate of ethanol metabolism, nor did sugars have a significant effect on reported hangover severity. Another study, examining the effectiveness of Morningfit, a hangover cure containing various B vitamins, showed that it did not significantly improve general well being during hangover [10]. Hence, it is unlikely that other ingredients mediate or prevent the effect of caffeinated beverages on alcohol hangover severity.

This study examined the relationship between the consumption of caffeinated beverages (energy drinks or colas) and alcohol during an evening of heavy drinking, and their effects on next day hangover severity.

\section{METHODS}

\section{Subjects and Recruitment}

Students were approached to complete a survey on drinking behavior at several locations such as the campuses, colleges and the library. They could choose to complete the survey immediately (paper/pencil), or online at a later moment. Previous studies showed that not all students consume alcohol, and that only part of the students that do use alcohol have experienced alcohol hangover [1]. Based on our previous experience, we aimed to approach approximately 1500 students to achieve an estimated sample size of about 500-600 students who experienced an alcohol 
hangover during the past year. Hence, no sampling method was applied to gather this convenience sample of subjects who experienced a hangover. The vast majority of students agreed to complete the survey. Subjects were included if they were over 18 years old, reported having had a hangover during the past month, and had used no drugs on that drinking occasion. Subjects were not compensated for their participation. The study was approved by the Institutional Review Board of Utrecht University, and the subjects remained anonymous.

\section{Survey Content}

Demographics, weekly alcohol consumption and other drinking variables were recorded, as well as a detailed investigation of all beverages that were consumed during the drinking session that produced their latest alcohol hangover. Hangover symptoms were scored using the Acute Hangover Scale [11]. Subjects could rate the severity of 8 hangover symptoms on a 10 -point scale ranging from 0 (absent) to 10 (highest severity). The items include headache, nausea, heart racing, being tired, dizziness, stomach ache, thirst, and reduced appetite. The mean of the item scores was computed to generate an overall hangover score, ranging from 0 (no hangover) to 10 (maximum score on each item).

\section{Statistical Analysis}

In The Netherlands, alcoholic consumptions contain a standardized amount of alcohol (10g). Because (non)alcoholic drinks in The Netherlands have standardized sizes, an estimate could also be made of the amount of caffeine (mg) that was mixed with alcohol. Energy drink (typically $250 \mathrm{ml}$ Red Bull $\left.{ }^{\circledR}\right)$ contains $80 \mathrm{mg}$ caffeine, whereas colatype beverages $(200 \mathrm{ml})$ contain 20 to $30 \mathrm{mg}$ caffeine [12, 13]. Analysis of variance (ANOVA) was applied to compare those who consumed alcohol with and without caffeinated beverages. In addition, those who consumed alcohol together with an energy drink were compared to those who consumed alcohol together with a cola-type beverage.

\section{RESULTS}

A total of 1503 subjects completed the survey. About half of the subjects $(\mathrm{N}=787,52.4 \%)$ reported having had a hangover during the past month. A total of $\mathrm{N}=549$ subjects (177 men and 372 women) met all inclusion criteria. Mean (SD) age was 20.4 (3.5) years old. Demographics are summarized in Table $\mathbf{1 .}$

On the evening before their latest hangover, subjects consumed a Mean (SD) of 11.10 (6.2) alcoholic consumptions. Total caffeine $(\mathrm{mg})$ did not correlate significantly with total alcohol consumption $(\mathrm{r}=0.08$, $\mathrm{p}=0.052$ ). Those who consumed alcohol in combination with caffeinated beverages did not consume significantly more alcohol $(p=0.111)$ than those who consumed alcohol without caffeinated beverages. Those who consumed alcohol together with an energy drink consumed less alcohol than those who drank alcohol alone, but the difference did not reach statistical significance $(p=0.056)$. In contrast, those who consumed alcohol together with a cola-type beverage consumed significantly more alcohol than those who consumed alcohol alone $(\mathrm{p}=0.001)$, or those who combined an energy drink with alcohol $(p=0.001)$ (See Fig. 1).

Scores on the hangover scale and the individual items are summarized in Table $\mathbf{2}$.

No significant differences were found in overall hangover severity or individual hangover symptoms on the Acute Hangover Scale between those who drink alcohol with or without caffeinated beverages. Also, no significant differences were found between overall hangover severity or individual symptoms between those who consumed energy drink and those who consumed cola beverages, even when taking differences in number of alcoholic consumptions into account.

\section{DISCUSSION}

The main finding of this study was that caffeinated beverages consumed together with alcohol did not reduce

Table 1. Demographics

\begin{tabular}{|c|c|c|c|c|c|}
\hline Demographics & $\begin{array}{l}\text { Overall } \\
N=549\end{array}$ & $\begin{array}{c}\text { Non-CAB } \\
\mathbf{N}=\mathbf{4 8 0}\end{array}$ & $\begin{array}{l}\text { CAB } \\
N=69\end{array}$ & $\begin{array}{l}\text { Colas } \\
N=45\end{array}$ & $\begin{array}{c}\text { Energy Drink } \\
\qquad \mathbf{N}=\mathbf{2 4}\end{array}$ \\
\hline Gender $(\mathrm{m} / \mathrm{f})$ & $177 / 372$ & $159 / 321$ & $18 / 51$ & $18 / 27$ & $0 / 24$ \\
\hline Age (years) & $20.4(3.5)$ & $20.4(3.6)$ & $20.5(2.9)$ & $20.4(2.5)$ & $20.6(3.5)$ \\
\hline BMI & $21.7(2.3)$ & $21.6(2.2)$ & $22.0(2.7)$ & $22.0(2.7)$ & $22.1(2.9)$ \\
\hline Alcohol units/week & $17.9(15.6)$ & $17.7(15.0)$ & $19.4(19.3)$ & $14.1(9.6)$ & $22.3(22.4)$ \\
\hline Hangovers / month & $2.6(2.4)$ & $2.6(2.2)$ & $2.7(3.3)$ & $3.0(3.8)$ & $2.1(1.7)$ \\
\hline Alcohol units consumed before the hangover & $11.1(6.2)$ & $10.9(6.1)$ & $12.2(7.1)$ & $14.2(7.6)$ & $8.5(4.2)^{*}$ \\
\hline Caffeine (mg) consumed before the hangover & $14.7(55)$ & 0 & $117.1(112)$ & $80.0(66)$ & $196.7(144)^{*}$ \\
\hline Sleep time (h, min.) & $6.27(2.0)$ & $6.25(2.1)$ & $6.38(1.9)$ & $6.41(1.8)$ & $6.33(2.2)$ \\
\hline
\end{tabular}

Mean (SD) are shown. Significant differences $(\mathrm{p}<0.05)$ between energy drink and cola drinkers are indicated by *. Other groups $(\mathrm{e} . \mathrm{g}$. , CAB versus non-CAB) did not differ significantly from each other. Abbreviations: $\mathrm{CAB}=$ caffeinated alcoholic beverages, $\mathrm{m}=$ male, $\mathrm{f}=$ female, $\mathrm{h}=$ hour, $\min .=$ minutes. 


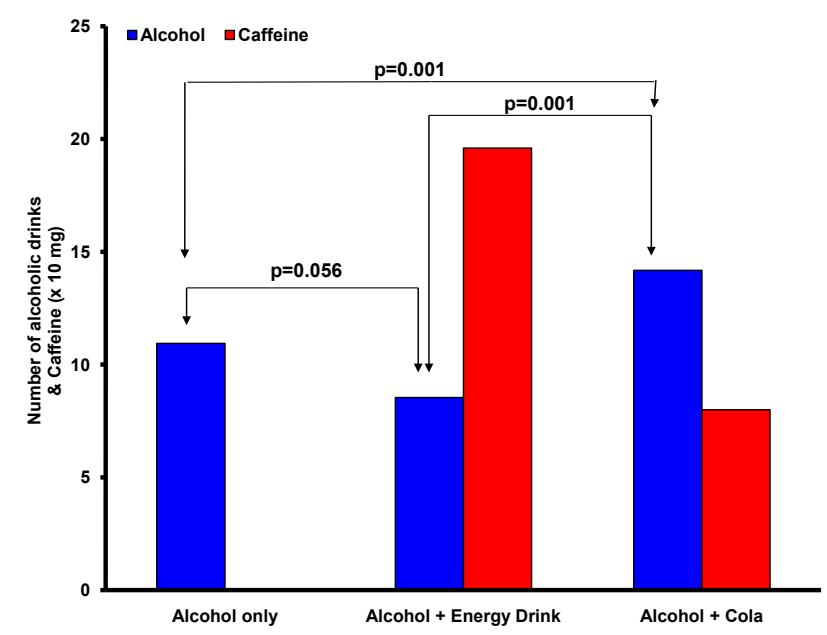

Fig. (1). Mean number of alcoholic consumptions and their total caffeine content. Differences are significant if $\mathrm{p}<0.05$.

alcohol hangover severity. The absence of a significant correlation between caffeine consumption and hangover severity was not surprising. Given the relative short half life of caffeine, it was not expected that potential effects of caffeine would persist during the hangover phase. No effect of mixing with caffeinated beverages was found on any individual hangover symptom, and the total score on the scale. Also, after adjusting for the total amount of alcohol that was consumed, no significant differences in hangover severity were found between those who mixed alcohol with energy drinks and those who mixed alcohol with cola beverages. A possible explanation is that the relative low doses of caffeine contained in caffeinated beverages are unable to counteract the effects of the relative high blood alcohol concentration (BAC) achieved on an evening of heavy drinking. A recent study by Howland et al. [14] tested participants after administering a relative high dose of alcohol $(\mathrm{BAC}=0.12 \%)$. They observed no significant differences between caffeinated beer and alcohol without caffeine on performance in a driving simulator. Participants did not report significant differences in the subjective effects of both beverages, such as perceived level of intoxication. The absence of caffeine effects at higher BAC found in their study may be an indication that also no differences will be observed during the subsequent hangover state. Regarding alcohol consumption, those who consumed alcohol together with a cola beverage consumed about $30 \%$ more alcohol on an evening of heavy drinking (14.2 units) than those who consumed alcohol with energy drinks (8.5 units), or those who consume alcohol without an energy drink (10.9 units).

A weakness of surveys is that the data presented are associations. This implies that the data do not provide evidence for the presence or absence of a cause-and-effect relationship between consumption of caffeinated beverages and alcohol consumption, or its hangover effects [15]. Double blind, experimental studies are needed to provide information on how to interpret the associations found in our survey, and others who examined this topic. Since we only questioned subjects about their last hangover episode and corresponding drinking behavior, it was not possible to evaluate whether caffeine consumption is associated with less frequent occurrence of hangovers. Another limitation concerns the small sample size of the subsamples.

Therefore, our data should be regarded as preliminary and future studies with larger sample sizes should confirm our findings. Preferably, such a survey would be conducted in different regions of the world, enabling cross-cultural comparisons to be undertaken for alcohol consumption of drinks with or without caffeinated beverages. It would also be interesting to investigate age and sex differences on hangover symptoms. Although caffeine consumption was not associated with hangover severity in our study, there is a need for experimental research confirming the effects of alcohol mixed with caffeinated beverages on hangover severity.

Table 2. Hangover Symptoms and their Severity

\begin{tabular}{|c|c|c|c|c|c|}
\hline Symptoms & $\begin{array}{c}\text { Overall } \\
N=549\end{array}$ & $\begin{array}{c}\text { Non-CAB } \\
\mathbf{N}=\mathbf{4 8 0}\end{array}$ & $\begin{array}{l}C A B \\
N=69\end{array}$ & $\begin{array}{l}\text { Colas } \\
N=45\end{array}$ & $\begin{array}{c}\begin{array}{c}\text { Energy Drink } \\
\mathbf{N}=\mathbf{2 4}\end{array}\end{array}$ \\
\hline Headache & $5.2(3.0)$ & $5.2(3.0)$ & $5.6(2.8)$ & $5.9(2.7)$ & $5.1(2.8)$ \\
\hline Nausea & $4.8(3.3)$ & $4.8(3.3)$ & $5.0(3.4)$ & $5.3(3.5)$ & $4.5(3.2)$ \\
\hline Being tired & $6.5(2.6)$ & $6.5(2.5)$ & $6.1(2.9)$ & $6.1(2.9)$ & $6.2(2.9)$ \\
\hline Headache & $5.2(3.0)$ & $5.2(3.0)$ & $5.6(2.8)$ & $5.9(2.7)$ & $5.1(2.8)$ \\
\hline Thirst & $5.8(3.1)$ & $5.8(3.0)$ & $6.0(3.2)$ & $6.3(3.3)$ & $5.6(3.0)$ \\
\hline Heart racing & $0.8(2.0)$ & $0.9(2.0)$ & $0.5(1.5)$ & $0.6(1.6)$ & $0.3(1.5)$ \\
\hline Dizziness & $2.1(2.8)$ & $2.1(2.8)$ & $2.1(3.0)$ & $2.0(2.9)$ & $2.4(3.2)$ \\
\hline Stomach ache & $2.0(2.8)$ & $2.0(2.8)$ & $1.6(2.6)$ & $1.6(2.5)$ & $1.6(2.8)$ \\
\hline Reduced appetite & $3.4(3.5)$ & $3.3(3.5)$ & $3.8(3.5)$ & $4.3(3.3)$ & $2.8(3.7)$ \\
\hline Total hangover score & $3.8(1.7)$ & $3.8(1.7)$ & $3.8(1.8)$ & $4.0(1.7)$ & $3.5(2.0)$ \\
\hline
\end{tabular}

Hangover symptoms are rated on a 10-point scale ranging from 0 (absent) to 10 (highest severity). CAB = Caffeinated alcoholic beverages. Scores did not differ significantly $(\mathrm{p}<0.05)$ between the groups. 


\section{ACKNOWLEDGEMENTS}

None declared.

\section{DISCLOSURE OF INTERESTS}

The authors report no conflicts of interest. The study was funded by Utrecht University. Over the past 3 years, Joris Verster has received research funding from Takeda Pharmaceuticals and Red Bull GmbH, and was scientific advisor for Takeda, Sanofi-Aventis, Transcept, Sepracor, Red Bull GmbH, Deenox, and CBD. These companies were not involved in the design and conductance of the study, analysis and interpretation of the data, and preparation of the manuscript.

\section{REFERENCES}

[1] Verster JC, Van Herwijnen J, Olivier B, Kahler CW. Validation of the Dutch Brief Young Adult Alcohol Consequences Questionnaire (B-YAACQ). Addict Behav 2009; 34: 411-4.

[2] Penning R, van Nuland M, Fliervoet LAL, Olivier B, Verster JC. The pathology of alcohol hangover. Curr Drug Abuse Rev 2010; 3:68-75.

[3] Gjerde H, Christophersen AS, Moan IS, et al. Use of alcohol and drugs by Norwegian employees: a pilot study using questionnaires and analysis of oral fluid. J Occup Med Toxicol 2010; 5: 13.

[4] Verster JC, Stephens R. The importance of raising the profile of alcohol hangover research. Curr Drug Abuse Rev 2010; 3: 64-7.

[5] Verster JC, Penning R. Treatment and prevention of alcohol hangover. Curr Drug Abuse Rev 2010; 3: 103-9.
[6] Pittler MH, Verster JC, Ernst E. Interventions for preventing or treating alcohol hangover: systematic review of randomized trials. BMJ 2005; 331:1515-8.

[7] Malinauskas BM, Aeby VG, Overton RF, Carpenter-Aeby T, Barber-Heidal K. A survey of energy drink consumption patterns among college students. Nutr J 2007; 6: 35 .

[8] O'Brien MC, McCoy TP, Rhodes SD, Wagoner A, Wolfson M. Caffeinated cocktails: energy drink consumption, high-risk drinking, and alcohol-related consequences among college students. Acad Emerg Med 2008; 15: 453-60.

[9] Ylikahri RH, Leino T, Huttunen MO, Pösõ AR, Eriksson CJP, Nikkilä EA. Effects of fructose and glucose on ethanol-induced metabolic changes and on the intensity of alcohol intoxication and hangover. Eur J Clin Invest 1976; 6: 93-102.

[10] Laas I. A double-blind placebo-controlled study on the effects of Morning Fit on hangover symptoms after a high level of alcohol consumption in healthy volunteers. J Clin Res 1999; 2: 9-15.

[11] Rohsenow DJ, Howland J, Minsky SJ, Greece J, Almeida A, Roehrs T. The acute hangover scale: A new measure of immediate hangover symptoms. Addict Behav 2007; 32: 1314-20.

[12] Reissig CJ, Strain EC, Griffiths RR. Caffeinated energy drinks - a growing problem. Drug Alcohol Depend 2009; 99:1-10

[13] International Food Information Council Foundation. IFIC Review: Caffeine and health: clarifying the controversies. Washington DC; USA 1998.

[14] Howland J, Rohsenow DJ, Arnedt JT, et al. The acute effects of caffeinated versus non-caffeinated alcoholic beverage on driving performance and attention/reaction time. Addiction 2011; 106: 33541.

[15] Verster JC, Alford C. Unjustified concerns about energy drinks Curr Drug Abuse Rev 2011; 4: 1-3. 